\title{
Kinetic Alfvén waves and plasma transport at the magnetopause
}

\author{
Jay R. Johnson and C. Z. Cheng \\ Princeton Plasma Physics Laboratory, Princeton, NJ 08543
}

\begin{abstract}
Large amplitude compressional type waves, with frequencies ranging from $10-500 \mathrm{mHz}$, are nearly always found in the magnetosheath near the magnetopause where there are large gradients in density, pressure and magnetic field. As compressional waves propagate to the magnetopause, these gradients efficiently couple them with shear/kinetic Alfvén waves near the Alfvén field-line resonance location $\left(\omega=k_{\|} v_{A}\right)$. We present a solution of the kinetic-MHD wave equations for this process using a realistic equilibrium profile including full ion Larmor radius effects and wave-particle resonance interactions for electrons and ions to model the dissipation. For northward IMF a KAW propagates backward to the magnetosheath. For southward IMF the wave remains in the magnetopause but can propagate through the $k_{\|}=0$ location. The quasi-linear theory predicts that KAWs produce plasma transport with a diffusion coefficient $D_{\perp} \sim 10^{9} \mathrm{~m}^{2} / \mathrm{s}$ and plasma convection on the order of $1 \mathrm{~km} / \mathrm{s}$. However, for southward IMF additional transport can occur because magnetic islands form at the $k_{\|}=0$ location. Due to the broadband nature of the observed waves these islands can overlap leading to stochastic transport which is much larger than that due to quasilinear effects.
\end{abstract}

\section{INTRODUCTION}

Wave observations in the magnetosheath and magnetopause indicated that ultra lowfrequency (ULF) waves with frequency less than $1 \mathrm{~Hz}$ are the dominant fluctuations. How- 


\section{DISCLAIMER}

This report was prepared as an account of work sponsored by an agency of the United States Government. Neither the United States Government nor any agency thereof, nor any of their employees, make any warranty, express or implied, or assumes any legal liability or responsibility for the accuracy, completeness, or usefulness of any information, apparatus, product, or process disclosed, or represents that its use would not infringe privately owned rights. Reference herein to any specific commercial product, process, or service by trade name, trademark, manufacturer, or otherwise does not necessarily constitute or imply its endorsement, recommendation, or favoring by the United States Government or any agency thereof. The views and opinions of authors expressed herein do not necessarily state or reflect those of the United States Government or any agency thereof. 


\section{DISCLAIMER}

Portions of this document may be illegible in electronic image products. Images are produced from the best available original document. 
ever, the origin of these ULF waves at the magnetopause remains an ongoing issue. In the magnetosheath substantial ULF compressional wave activity exists nearly all of the time, and under most conditions the power level of wave activity is usually 10-100 times larger than the wave activity in the solar wind or the magnetosphere [1]. Specific magnetopause crossings have been studied for both northward $[2,3]$ and southward $[4,5]$ IMF conditions. Interestingly enough, ULF wave activity in the magnetopause exhibits strikingly different characteristics than waves observed at the magnetosheath: magnetosheath waves are primarily compressional while waves at the same frequency range at the magnetopause are transverse and often have larger amplitude. For southward IMF the fluctuation level of the transverse waves at the magnetopause are typically enhanced by a factor of 10 over the level of the compressional waves in the magnetosheath [4]. These observations led [6] to suggest that coupling between the compressional waves and the Alfvén resonance based on ideal MHD theory was responsible for the observed wave signatures. However, until now there has been no satisfactory theory for these wave observations. In this paper we present the theory of mode conversion of compressional waves into kinetic Alfvén waves (KAWs) at the magnetopause to explain these observed wave features at the magnetopause.

Presently, it seems to be generally accepted that the various high frequency (larger than ion cyclotron frequency) waves cannot provide the necessary transport $[7,8]$ to explain the amount of solar wind plasma that enters the magnetosphere because the high frequency power spectrum near the magnetopause is weak and obeys a power law which falls off rapidly. An exception is the recent theory of [9] which indicated that the current convective instability with frequency in the lower-hybrid wave frequency range $(700 \mathrm{~Hz}-1 \mathrm{kHz})$ can lead to significant transport in the magnetopause current layer. On the other hand, there is a general conjecture that resistive MHD type magnetic reconnection (or merging) is the dominant plasma transport mechanism at the magnetopause, although no comprehensive theoretical study has been performed. However, ULF transverse waves are commonly observed with dominant wave power at the magnetopause [2,10-12], and plasma transport resulting from ULF waves has not been studied. It is well known [13] that in laboratory 
plasmas MHD waves are a leading cause of plasma transport, and the hybrid simulations of [14] indicated that low frequency drift-like waves with frequency below the ion cyclotron frequency can give rise to a diffusion coefficient comparable to the Bohm rate $\left(\sim 10^{9} \mathrm{~m}^{2} / \mathrm{s}\right)$ at the magnetopause. In this paper, we will also address transport which results from the observed ULF waves which have dominant wave power at the magnetopause.

Our proposed scenario for magnetopause wave activity is that compressional MHD waves in the magnetosheath propagate to the magnetopause and couple with transverse KAWs at the magnetopause. The coupling occurs where the compressional wave frequency, $\omega$, matches the local shear Alfvén frequency, $k_{\|} v_{A}$. The KAW exhibits two properties in contrast to the incoming compressional wave: its polarization is primarily transverse and its wave amplitude is strongly enhanced (by a factor of 10) over the level of the compressional waves in the magnetosheath.

The KAW has important implications for plasma transport. When the perpendicular wavelength is on the order of the gyroradius, ion motion decouples from the electron motion due to polarization drift effects leading to charge separation. Typically $v_{e}>\omega / k_{\|}>v_{i}$ so that electrons can maintain charge quasi-neutrality which provides a finite parallel electric field. Decoupling of the resonant ions from the magnetic field causes transport and these ions move across the magnetopause in only a few wave periods. The electrons follow due to ambipolar effects.

These wave and transport processes depend on the IMF direction. For northward IMF, the Alfvén frequency $\left(\omega_{A}=k_{\|} v_{A}\right)$ monotonically increases and field-line resonance occurs near the $\omega=\omega_{A}$ location. The wave amplitudes are largest near the resonance location and the KAW radiates back into the magnetosheath. Assuming random phase nature of the broadband KAWs, transport due to KAWs will scale with the quasilinear theory result. For southward IMF, magnetic shear in the current layer gives rise to $k_{\|}=0$ locations as illustrated in Figure 1 where KAWs form magnetic islands. Because the wave spectrum is broadband, it is likely that many such islands form at different locations and overlap. Overlapping of particle phase space islands leads to interlinking particle flux which yields 


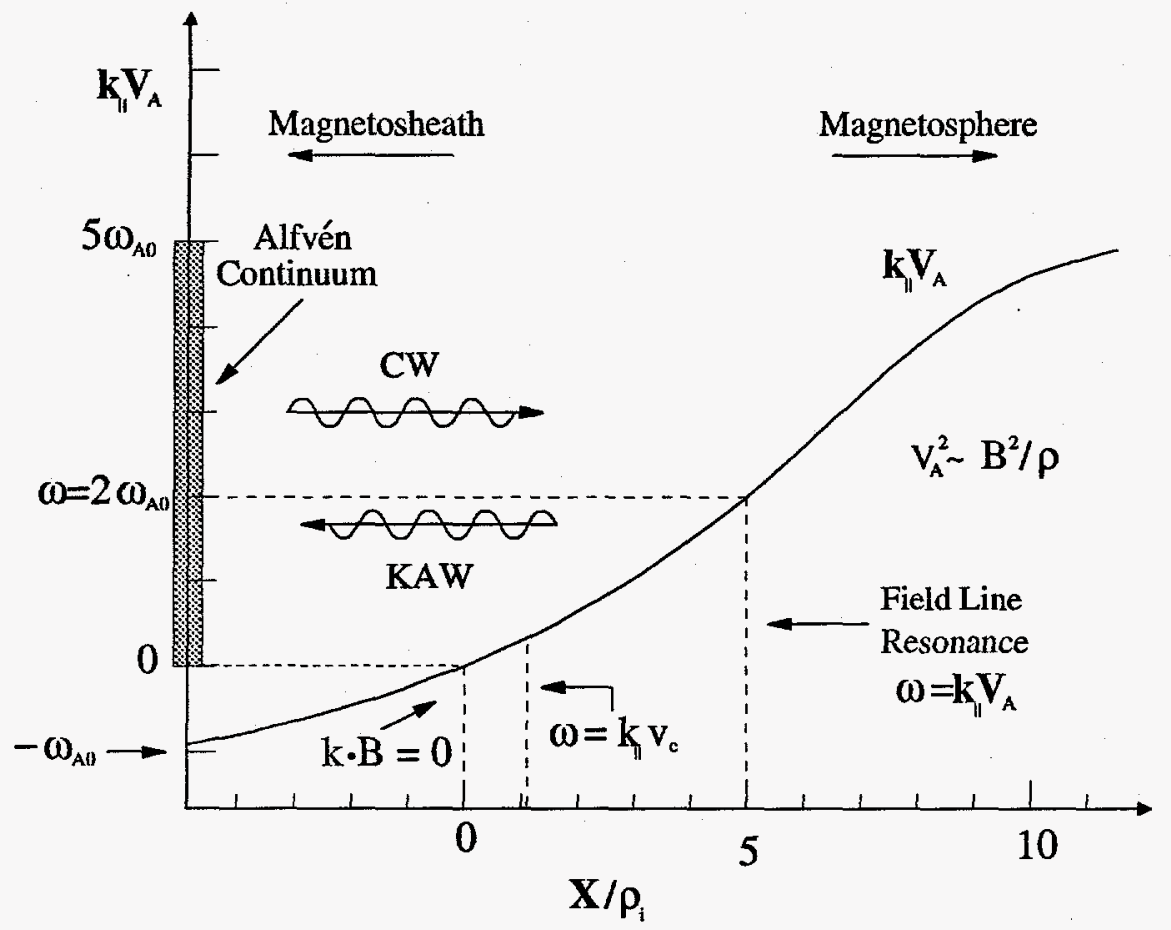

FIG. 1. Alfvén frequency for a typical magnetopause crossing from the magnetosheath to the magnetosphere for southward IMF. The position is shown scaled to $\rho_{i}$, and the magnetopause scale length is $10 \rho_{i}$. The Alfvén velocity is enhanced by a factor of 10 across the magnetopause. Compressional type waves (CW) with frequency $\omega$ propagate to the field line resonance location $\left(\omega=k_{\|} v_{A}\right.$ at $\left.x=5 \rho_{i}\right)$ and mode convert to backward propagating kinetic Alfvén waves. Because of the magnetic drift effects, KAWs can propagate to the $k_{\|}=0$ location $(x=0)$ and drive time-dependent (patchy) magnetic reconnection.

massive transport well above the quasilinear level and causes local flattening of plasma profiles.

\section{MODEL FOR WAVE ACTIVITY AT THE MAGNETOPAUSE}

General magnetopause features of the background magnetic field, density, flow, and temperature have been cataloged by the surveys of [15] for both high and low magnetic shear. In both cases background gradients are large and the Alfvén velocity can increase across the magnetopause by about a factor of 10. For a typical parallel wavelength of 1 
$\mathrm{R}_{\mathrm{E}}$ and $400 \mathrm{~km} / \mathrm{s}<v_{A}<4000 \mathrm{~km} / \mathrm{s}$ at the magnetopause, the Alfvén field line resonance frequency forms a continuous spectrum and spans the range $60 \mathrm{mHz}<f<600 \mathrm{mHz}$ which overlaps with the frequency band with maximum wave power in the magnetosheath. Near the resonance location, the compressional wave couples strongly with the shear Alfvén wave which has a logarithmic singularity in transverse wave power according to the MHD theory. However, [16] showed that this resonance singularity may be resolved by retaining the ion gyroradius effects.

We investigate the above scenario using the kinetic-MHD model [17]. This model incorporates kinetic effects into the MHD equations using Ohm's law and momentum equation obtained from the gyrokinetic equation. The essential approximations required by this model are that $\omega \ll \Omega_{i}, k_{\perp} L_{\perp} \gg 1, k_{\|} L_{\|} \gg 1$, and $\rho_{i} \ll L_{\perp}$, where $\rho_{i}=v_{i} / \Omega_{i}, v_{i}^{2}=T_{i} / m_{i}, \Omega_{i}$ is the ion cyclotron frequency, and $L_{\perp, \|}$ are the magnetopause perpendicular and parallel equilibrium scale lengths, respectively. At the magnetopause, $\rho_{i} \sim 50 \mathrm{~km}, L_{\perp} \sim 10 \rho_{i}$ and $L_{\|} \sim 10 \mathrm{R}_{\mathrm{E}}$ so that the approximation is typically quite good.

This model is appropriate for studying this problem because it: (a) includes full Larmor radius effects for ions, (b) includes diamagnetic, $\nabla B$, and curvature drifts, (c) is valid in a high $\beta$ plasma, and (d) includes wave-particle resonance. The ion Larmor radius effects are essential in describing mode conversion of MHD waves into KAWs. Because $k_{\perp} \rho_{i} \sim 1$, full ion Larmor radius effects must be included. Diamagnetic and magnetic drift frequencies can be on the order of the Alfvén frequency and their effects must be included in the region of large plasma and magnetic field gradient. Wave particle resonance is very important for two reasons. First, the quasi-linear transport occurs due to the waves results from wave particle resonance, and second, electron Landau damping can be quite large when the wave phase velocity becomes on the order of the electron thermal velocity and will occur as $k_{\|} \rightarrow 0$ for southward IMF. The magnetic drift contributes to the resonance process and can shift the electron Landau damping location so that the KAW propagates to the $k_{\|}=0$ location.

For simplicity, we take a one-dimensional model with variation along the radial direction $x$ from the magnetosheath to the magnetopause. We employ the Padé approximation, 
$I_{0}(b) e^{-b} \approx(1+b)^{-1}$ where $b=k_{\perp}^{2} \rho_{i}^{2}$, so that our analysis is a good approximation for studying full ion Larmor radius effects. Given these approximations, the wave equation which couples the KAW with the compressional wave may be obtained from [17] and is given by

$$
\begin{gathered}
k_{\|}^{2} v_{A}^{2}(1+\Delta) \rho_{i}^{2} \frac{d^{4} \phi}{d x^{4}}+\frac{d}{d x}\left(\tilde{\omega}^{2}-k_{\|}^{2} v_{A}^{2}\right) \frac{d \phi}{d x}+ \\
\left(\tilde{\omega}^{2}-k_{\|}^{2} v_{A}^{2}\right)\left(\tilde{\omega}^{2}-k_{y}^{2} v_{A}^{2}-k_{\|}^{2} v_{A}^{2}\right) \phi / v_{A}^{2}=0
\end{gathered}
$$

where $\tilde{\omega}^{2}=\omega\left(\omega-\omega_{\star i}\right)$ where $\omega_{\star i}=\mathbf{k} \cdot \mathbf{b} \times \nabla \ln P_{i} / \Omega_{i}$ is the ion diamagnetic drift frequency

$$
\begin{gathered}
\Delta=\frac{T_{e}\left(1-\omega_{\star i} / \omega\right)}{T_{i} W_{e}+T_{e} W_{i}} \\
W_{e, i}=\frac{T}{n} \int d^{3} v \frac{\partial F}{\partial \mathcal{E}} \frac{\left(\omega-\omega_{\star T}\right) k_{\|} v_{\|} J_{0}^{2}\left(k_{\perp} v_{\perp} / \Omega\right)}{\omega\left(\omega-k_{\|} v_{\|}-\omega_{d}\right)}
\end{gathered}
$$

where $\left.\omega_{\star T}=\mathbf{k} \cdot \mathbf{b} \times \nabla F /(\Omega \partial F / \partial \mathcal{E})\right]$, and $\omega_{d}=\mathbf{k} \cdot(\mathbf{b} / \Omega) \times\left[\left(v_{\perp}^{2} / 2\right) \nabla \ln B+v_{\|}^{2} \boldsymbol{\kappa}\right]$ is the magnetic drift frequency. Note that in obtaining numerical values of $W$, we have neglected the secondary effects due to particle curvature drift and Larmor radius effects.

We solve Eq. (1) using asymptotic analysis. When far away from the resonance, there are two spatial scales which characterize the solution, an MHD scale which is the balance of the second and third terms of Eq. (1),

$$
\begin{aligned}
& v_{A}^{2} \frac{d}{d x}\left(\tilde{\omega}^{2}-k_{\|}^{2} v_{A}^{2}\right) \frac{d \phi}{d x}+ \\
& \quad\left(\tilde{\omega}^{2}-k_{\|}^{2} v_{A}^{2}\right)\left(\tilde{\omega}^{2}-k_{y}^{2} v_{A}^{2}-k_{\|}^{2} v_{A}^{2}\right) \phi=0,
\end{aligned}
$$

and a kinetic scale which is the balance of the first and second terms of Eq. (1)

$$
\rho_{i}^{2}(1+\Delta) \frac{d^{2} \phi}{d x^{2}}+\left(\tilde{\omega}^{2} / k_{\|}^{2} v_{A}^{2}-1\right) \phi=0
$$

In this regime, the solution consists of a linear superposition of four independent solutions (two for each of the above equations). Near the Alfvén resonance (at $x_{0}$ ) an inner equation can be found by linearizing $\tilde{\omega}^{2}-k_{\|}^{2} v_{A}^{2} \approx-\tilde{\omega}_{0}^{2}\left(\bar{x} / L_{B}\right)\left(1+\mathcal{O}\left(\bar{x} / L_{B}\right)^{2}\right)$ where $\bar{x}=\left(x-x_{0}\right)$. 
The differential equation in terms of $X=\bar{x} / \delta$ with $\delta^{3}=\rho_{i}^{2} L_{B}(1+\Delta)$, is the inhomogeneous Airy equation,

$$
\frac{d^{2}}{d X^{2}} \frac{d \phi}{d X}-X \frac{d \phi}{d X}=E_{0 x}\left(\frac{L_{B}}{\rho_{i}}\right)^{2 / 3}
$$

where $E_{0 x}$ is amplitude of the MHD wave, and has the well known solution [18]

$$
\delta E_{x}=\pi E_{0 x}\left(\frac{L_{B}}{\rho_{i}}\right)^{2 / 3}\left[c_{1} \operatorname{Ai}\left(\frac{\bar{x}}{\delta}\right)+c_{2} \operatorname{Bi}\left(\frac{\bar{x}}{\delta}\right)+\operatorname{Gi}\left(\frac{\bar{x}}{\delta}\right)\right]
$$

where $\mathrm{Ai}, \mathrm{Bi}$, and $\mathrm{Gi}$ are Airy functions defined in [19], $c_{1}$ and $c_{2}$ are determined by the matching to the outer WKB solutions. Note that there is a good overlap region for matching the inner solution, valid for $x<L_{B}$, with the outer solutions, valid for $x>\left(\rho_{i}^{2} L_{B} / 4\right)^{1 / 3}$ $\left(10 \gg\left|\bar{x} / \rho_{i}\right| \gg>1\right.$ for typical magnetopause parameters). We solve Eqs. (4) and (5) numerically and constrain the outer solution (which is a superposition of these two solutions) to have only an incoming MHD wave and no incoming KAW. The numerical match to the inner solution determines the coefficients, $c_{1}$ and $c_{2}$. It is to be noted that the relative amplitudes of the two outer solutions is $\left(\delta E_{K A W} / \delta E_{M S}\right)_{\text {out }} \approx \sqrt{\pi}\left(L_{B} / \rho_{i}\right)^{2 / 3} \xi \sim 10$ where $\xi=\mathcal{O}(1)$ is a complex numerical coefficient determined from the matching conditions.

\section{WAVE STRUCTURE AT THE MAGNETOPAUSE}

For weak magnetic shear at the magnetopause (northward IMF cases), Eq. (7) shows that a KAW will propagate backward towards the magnetosheath. The strong magnetic shear case is more interesting, and we perform numerical solutions of Eq. (1) for a model magnetopause equilibrium based on the observations of a southward IMF crossing by [10]. A typical profile for the Alfvén velocity in this model is shown in Figure 1. We choose the incoming compressional wave frequency satisfying the Alfvén field line resonance condition

at $x_{0}=5 \rho_{i}$. We also choose $k_{\|}=0$ to be at $x=0$. The gradient scale length of the magnetopause width is approximately $10 \rho_{i}$.

Figure 2 shows the numerical solutions of Eq. (1) in the outer region. The top two panels show the outer MHD solution for the electric field, $\delta E_{x}=-d \phi / d x$ which satisfies 


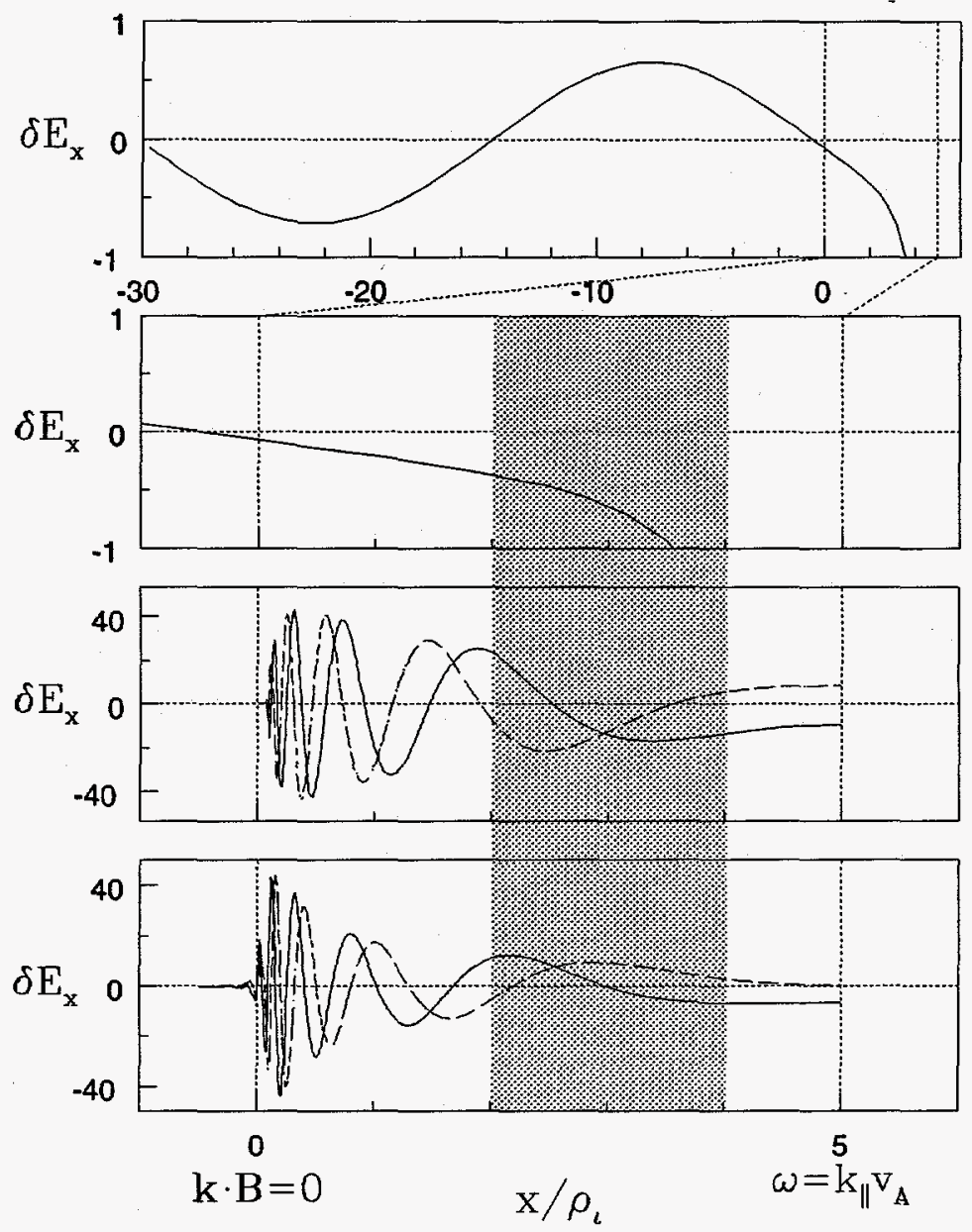

FIG. 2. The outer solutions of Eq. (1). The upper two panels show the same outer MHD solution for $\delta E_{x}$ which satisfies the boundary condition of an incoming compressional wave. The interesting region is between $x=0$ and $x=5$ indicated by the dashed lines in all panels. The third panel shows the kinetic Alfvén wave outer solution without including diamagnetic and magnetic drift effects. The dashed and dotted lines correspond to the real and imaginary parts of the solution. Notice that the wave propagates from the Alfvén resonance location $(x=5)$ toward the $k_{\|}=0$ location $(x=0)$ but is absorbed before getting there. because of strong electron Landau damping. The bottom panel shows the outer solution of KAW by including realistic magnetic drift effects with a nominal value of $\mathbf{k} \cdot \mathbf{v}_{d} / \omega \sim 1$ evaluated near at the maximum of $\mathbf{k} \times \mathbf{b} \cdot \hat{x}$. Notice that appreciable amplitude is found near the $k_{\|}=0$ location because magnetic drift effects shift the electron Landau damping location beyond the $k_{\|}=0$ location. The outer solutions are matched to the inner analytic solution in the shaded region. 
the boundary condition of an incoming compressional Alfvén wave. The solution behaves as $1 / x$ near the Alfvén resonance location. In the third panel we plot the KAW outer solution without including diamagnetic and $\nabla B$ effects. The dashed and dotted lines correspond to the real and imaginary parts of the KAW solution. Notice that the wave propagates from the Alfvén resonance location $\left(x=5 \rho_{i}\right)$ toward the $k_{\|}=0$ location $(x=0)$ but is absorbed at the electron damping point where $\omega=k_{\|} v_{e}$ before getting there. We also note that the peak KAW amplitude is about a factor of 40 larger than the outer MHD solution. In the bottom panel we take realistic drift effects with a nominal value of $\mathbf{k} \cdot \mathbf{v}_{d} / \omega \sim 1$ evaluated near at the maximum of $\mathbf{k} \times \mathbf{b} \cdot \hat{x}$. Notice that appreciable amplitude is found near the $k_{\|}=0$ location. The KAW propagates to the $k_{\|}=0$ location because the $\nabla B$ drift shifts the resonance and reduces electron Landau damping.

When broadband compressional waves propagate from the magnetosheath to the magnetopause, broadband transverse KAWs will be excited and distributed over the entire magnetopause with enhanced amplitude. We emphasize that the above numerical wave solutions are consistent with the observed wave features in the magnetosheath and magnetopause: transverse waves at the magnetopause are broadband and are strongly enhanced (by a factor of 10) over the level of the compressional waves in the magnetosheath.

\section{TRANSPORT RESULTING FROM KINETIC Alfvén WAVE ACTIVITY}

Based on the quasilinear theory, KAWs can cause substantial transport for both northward and southward IMF. From the gyrokinetic equation, we find that the density evolves according to

$$
\frac{\partial n}{\partial t}+\frac{\partial}{\partial x}\left(V_{c} n\right)=\frac{\partial}{\partial x} D_{\perp} \frac{\partial n}{\partial x}
$$

This quasilinear transport equation results from decoupling of the plasma from field lines due to both the parallel electric field as well as the perpendicular electric field that couples to the particle magnetic drift motion. For a Gaussian ion distribution function (assuming 
$\left.\omega>\omega_{d}\right)$, the transport coefficients are approximately

$$
D_{\perp} \sim \sum_{k} \sqrt{\frac{\pi}{\beta}} e^{-1 / \beta}\left(\frac{v_{A}}{\left|k_{\|}\right|}\right)\left[2\left(\frac{k_{y} \bar{v}_{d}}{k_{\|} v_{A}}\right)^{2}+\left(\frac{T_{e}}{T_{i}} k_{x}^{2} \rho_{i}^{2}\right)^{2}\right]\left|\frac{\delta B_{x k}}{B_{o}}\right|^{2}
$$

and

$$
V_{c} \sim-\sum_{k} \sqrt{8 \pi} \beta^{-1} e^{-1 / \beta} \bar{v}_{d}\left(k_{x} \rho_{i} \frac{T_{e} k_{x}}{T_{i}\left|k_{\|}\right|}\right)\left|\frac{\delta B_{x k}}{B_{o}}\right|^{2} .
$$

where $\bar{v}_{d}$ is the particle magnetic drift velocity evaluated with $v_{\perp}^{2}=2 T_{i} / m_{i}$. In Eq. (9) the first term is due to magnetic drift velocity coupling to the perpendicular electric field. The second term is due to parallel electric field and was previously derived by [20] and was used in the previous work by [21]. Note that at the magnetopause the diffusion is dominated by the first term associated with magnetic drift effect. Using typical magnetopause parameters, $T_{e} / T_{i} \sim 0.2, k_{x}^{2} \rho_{i}^{2} \sim 0.25, \lambda_{\|} \sim 1 \mathrm{R}_{\mathrm{E}}, \delta B_{x} / B_{o}=0.1$, and $v_{A}=500 \mathrm{~km} / \mathrm{s}$, and replacing the summation over the perturbed magnetic field with an average value, we find that the diffusion coefficient is $D_{\perp} \sim 10^{9} \mathrm{~m}^{2} / \mathrm{s}$ and the radial ion convection velocity is $V_{c} \sim 1 \mathrm{~km} / \mathrm{s}$. For southward $B_{0}, V_{c}$ is toward the magnetosphere, while for northward $B_{0}, V_{c}$ is toward the magnetosheath. For southward IMF the convection would tend to sharpen the density profile.

For southward IMF conditions, plasma transport can be further enhanced over the quasilinear prediction by magnetic reconnection resulting from KAWs. Reconnection can result from magnetic perturbations with finite radial amplitude at the location where $\mathbf{k} \cdot \mathbf{B}_{o}=k_{z} B_{z o}(x)+k_{y} B_{y o}(x)=0, \mathbf{k}$ is the wave vector. For a radial magnetic field perturbation with $\delta B_{x}=\delta \psi(x) \cos \left(k_{y} y+k_{z} z-\omega t\right)$, a magnetic island appears in the $x-s$ plane with a width $\Delta x \simeq\left(L_{s} \delta B_{x} / k_{y} B_{o}\right)^{1 / 2}$, where $s=y+k_{z} z / k_{y}, L_{s}=\left|d \ln \left(\mathbf{k} \cdot \mathbf{B}_{o}\right) / d x\right|^{-1}$ is the magnetic shear scale length. Because there is a broadband spectrum of compressional waves in the magnetosheath, KAWs are present at different $k_{\|}=0$ locations where magnetic islands form. Particle orbit islands, corresponding to the magnetic islands, will form in the particle phase space. When KAW fluctuation level is above a threshold value, particle orbit islands overlap, and particle orbits are stochastic. Massive particle transport can occur 
leading to flattening of plasma profiles at the magnetopause. Thus it would be expected that the character of the transport and thus the structure of the background magnetic field and density profiles for southward IMF cases would be strikingly different from northward IMF cases.

\section{SUMMARY}

In summary, we have presented a physical process of generating transverse Alfvén waves at the magnetopause: compressional waves propagating from the magnetosheath can mode convert into transverse KAWs at the magnetopause with fluctuation level enhanced over the incoming compressional wave level. We have shown that the radial structure of KAWs depends sensitively on the magnetic shear at the magnetopause. Moreover, quasi-linear theory predicts that KAWs can cause plasma transport with a diffusion coefficient $D_{\perp} \sim 10^{9} \mathrm{~m}^{2} / \mathrm{s}$ and a plasma convection on the order of $1 \mathrm{~km} / \mathrm{s}$. However, for southward IMF additional transport can occur because KAWs form magnetic islands at $k_{\|}=0$ locations. Due to the broadband nature of KAWs these islands can overlap leading to stochastic transport which is much larger than that due to quasilinear effects. Massive stochastic particle transport can lead to local flattening of the plasma density profile where islands overlap.

We emphasize that there are three particular features of wave observations in the magnetosheath and magnetopause that support the KAW scenario. First, most of the wavepower in the magnetosheath and near the magnetopause is at low frequencies $(10-500 \mathrm{mHz})$. Secondly, large gradients in the background plasma density, magnetic field, and pressure are observed at the magnetopause so that the Alfvén velocity can vary by up to a factor of 10 . Finally, large peaks in the $\delta B_{\perp}$ component are observed by the high resolution instrument

during magnetopause crossings while the analytical result predicts the KAW wave amplitude to be enhanced by an order of magnitude. As a further extension of this work, we plan to use the compressional wave frequency and wavenumber spectrum observed in the magnetosheath near the magnetopause to obtain the radial solutions of KAWs at the mag- 
netopause by linear superposition of all KAWs corresponding to each frequency and wave number which we can rigorously compare with satellite observations.

Finally, we conclude by pointing out that quantitative studies of plasma transport due to KAWs are yet to be performed. In addition, plasma transport due to competing mechanisms such as resistive magnetic reconnection (or merging) has not been performed and must also be addressed before a conclusion can be drawn on the transport processes at the magnetopause.

\section{ACKNOWLEDGMENTS}

This work is supported by the NSF grant ATM-9523331 and DoE Contract No. DEAC02-76-CH03073. 


\section{REFERENCES}

[1] B. J. Anderson, in Physics of the Magnetopause, Geophysical Monograph Series (American Geophysical Union, Washington, D. C., 1995), Vol. 90.

[2] P. Song, C. T. Russell, and C. Y. Huang, J. Geophys. Res. 98, 5907 (1993).

[3] X. X. Zhang et al., Geophys. Res. Lett. 23, 105 (1996).

[4] L. Rezeau, S. Perraut, and A. Roux, Geophys. Res. Lett. 13, 1093 (1986).

[5] P. Song and C. T. Russell, J. Geophys. Res. 97, 1411 (1992).

[6] G. Belmont, F. Reberac, and L. Rezeau, Geophys. Res. Lett. 22, 295 (1995).

[7] J. LaBelle and R. A. Treumann, Space. Sci. Rev. 45, 175 (1988).

[8] R. Treumann, J. Labelle, and T. M. Bauer, in Physics of the Magnetopause, Geophysical Monograph Series (American Geophysical Union, Washington, D. C., 1994), Vol. 90.

[9] J. F. Drake, J. Gerber, and R. G. Kleva, J. Geophys. Res. 99, 11211 (1994).

[10] P. Song et al., J. Geophys. Res. 98, 187 (1993).

[11] L. Rezeau, A. Morane, S. Perraut, and A. Roux, J. Geophys. Res. 94, 101 (1989).

[12] L. Rezeau, A. Roux, and C. T. Russell, J. Geophys. Res. 98, 179 (1993).

[13] C. Z. Cheng et al., in Plasma Physics and Controlled Nuclear Fusion Research 1994, Vol. 3 (International Atomic Energy Agency, Vienna, 1996), pp. 373-389.

[14] D. Winske and N. Omidi, J. Geophys. Res. 100, 11,923 (1995).

[15] G. Paschmann, W. Baumjohann, N. Sckopke, and T. D. Phan, J. Geophys. Res. 98, 13,409 (1993).

[16] A. Hasegawa, J. Geophys. Res. 81, 5083 (1976).

[17] C. Z. Cheng, J. Geophys. Res. 96, 21,159 (1991). 
[18] A. Hasegawa and L. Chen, Phys. Fluids 19, 1924 (1976).

[19] M. Abramowitz and I. Stegun, Handbook of Mathematical Functions (Dover Publishing Co., New York, NY, 1970).

[20] A. Hasegawa and K. Mima, Phys. Rev. Lett. 36, 1362 (1978).

[21] L. C. Lee, J. R. Johnson, and Z. W. Ma, J. Geophys. Res. 99, 17405 (1994). 